\title{
MAGNETIC DOMAIN DYNAMICS IN FeTb THIN FILMS
}

\author{
BAKURI LANCHAVA, KOJI MATSUMOTO ${ }^{\dagger}$ and HORST HOFFMANN
}

\author{
Institut für Angewandte Physik, Universität Regensburg, Universitätstr. 31, 93053 Regensburg, Germany

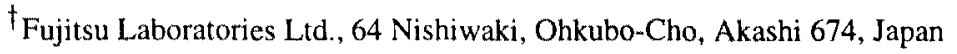

\begin{abstract}
Magnetic domain patterns and their dynamics in $500 \AA$ thick $\mathrm{Fe}_{1-\mathrm{x}} \mathrm{Tb}_{\mathrm{x}}$ amorphous magnetron-sputtered films with $0.16<x<0.35$ have been studied experimentally. All films possess a strong perpendicular anisotropy (PMA) with the anisotropy constant $K_{U} \approx 3 \cdot 10^{6} \mathrm{erg} / \mathrm{cm}^{3}$. For the observation of the magnetic domains a Kerr microscopic technique was used. The Kerrmicroscopical observations showed that the domain patterns are strongly dependent on the film composition. It was also seen that the deviation of the composition from the compensation point $(x \approx 0.23$ ) plays a decisive role in the pattern appearance. The time dependence of the integral Kerr amplitude gives the temporal evolution of the remagnetization process. In order to describe the experimental observations a micromagnetic model was developed showing good agreement with the experimental results.
\end{abstract}

KEYWORDS; Fe-Tb, AMORPHOUS, PERPENDICULAR ANISOTROPY, MAGNETIC DOMAINS, MAGNETIZATION REVERSAL, MICROMAGNETICS

\section{INTRODUCTION}

The growing interest of scientists and engineers in amorphous $\mathrm{Fe}-\mathrm{Tb}$ thin films is connected with the discovery of perpendicular magnetic anisotropy (PMA), which enables one to use them for magneto-optical (MO) data storage. Although the MO media are already successfully manufactured and used for data storage the physical limitations of this promising new technology are not yet well understood [1].

In this paper magnetic properties of argon-sputtered $\mathrm{Fe}_{1-\mathrm{x}} \mathrm{Tb}_{\mathrm{x}}$ film system are studied experimentally and the magnetic domains in these films are observed by means of a Kerr microscope described elsewhere [2].

It turned out that the domain patterns are directly correlated with the value of saturation magnetization $M_{S}$ in both iron-rich and terbium-rich regions. In the vicinity of the compensation point $(x \approx 0.23$ at room temperature) the domain patterns are more circular, while away from this point the stripe domains appear. The time dependence of the Kerr signal amplitude integrated from the entire film surface under observation gives the temporal evolution of the remagnetization process.

In order to describe the experimentally observed domain patterns or correlations between the magnetization states at different sites of the sample a micromagnetic model on a hexagonal grid was developed, leading to an "equation of motion", which can be formally connected with the Monte Carlo algorithm with an Ising-like Hamiltonian. In addition an effective exchange field between the neighboring cells is introduced. The efficiency of this method for calculation of domain patterns have been already demonstrated [3]. The Fast Fourier Transformation (FFT) algorithm implemented here yields additionally a powerful tool for calculating the spatial distribution of the demagnetizing field $H_{d}$ with periodic boundary conditions $[1,4]$.

\section{EXPERIMENTAL}

The amorphous $\mathrm{Fe}_{1-\mathrm{x}} \mathrm{Tb}_{\mathrm{x}}$ films were manufactured by rf-sputter-deposition on glass substrates. Sputtering was carried out in an argon atmosphere at a pressure of $5 \cdot 10^{-3}$ mbar. As target materials mosaic $\mathrm{Fe}-\mathrm{Tb}$ targets were used in order to get films with variable composition. The nominal thickness of the FeTb layer was equal to $50 \mathrm{~nm}$. The actual thicknesses were controlled by X-ray fluorescence.

For the magnetic characterization of the samples a vibration sample magnetometer (VSM) and a torque magnetometer were used. The films under studies showed ferrimagnetic behavior in the whole range of the compositional parameter $(0.16<x<0.35)$.

The VSM measurements showed an almost symmetrical dependence of the saturation magnetization $M_{S}$ on the compositional parameter $x$ on both sides from the compensation point ( $x=0.23$ ) with $M_{S} \approx 250 \mathrm{G}$ at $x=0.16$ and at $x=0.3$. The torque measurements revealed a strong PMA in all the samples with the anisotropy constant $K_{U} \approx 3 \cdot 10^{6}$ $\mathrm{erg} / \mathrm{cm}^{3}$. From the measured value of $K_{U}$ and the literature value of the exchange stiffness $A$ [5] the domain wall width $\delta_{W}$ can be estimated. For the investigated films we $\operatorname{got} \delta_{W} \approx 100 \AA$.

For the observation of magnetic domains a Kerr microscope with a real-time digital image processing, described previously [2], was used. The domains become observable by means of the polar Kerr effect, allowing us to study the magnetization component perpendicular to the film plane. The magnetization state of a sample can be changed during the observations by applying external magnetic fields. A special coil under the microscope objective allowed us to create perpendicular magnetic fields up to $12 \mathrm{kOe}$. An additional low inductance coil with the maximal field of some 


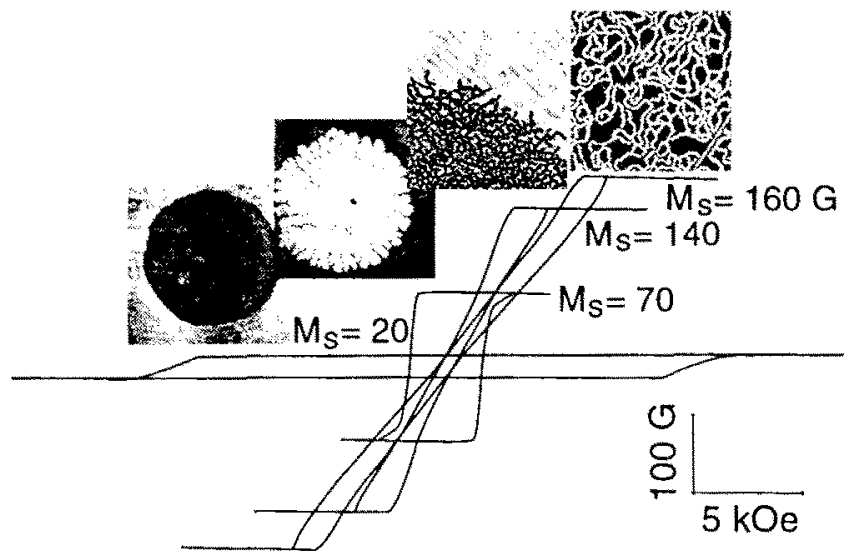

Figure 1: Hysteresis loops and domain patterns observed experimentally by means of the polar Kerr effect. The dark and light areas correspond to the domains with opposite magnetization direction. domain observation field is restricted to $70 \mu \mathrm{m} \times 70 \mu \mathrm{m}$.

200 Oe was used to trigger such irreversible dynamic processes as domain wall motion or creation of nuclei during the magnetization reversal.

The observed correlation between the domain patterns, measured hysteresis loops and the value of $M_{S}$ is shown in Fig. 1. In dependence on the value of saturation magnetization four main domain patterns were observed. The "cauliflower" domain (second from left in the figure) appears in a narrow range of composition somewhat apart from the compensation point.

The time-dependent measurements of the magnetiza tion reversal together with the domain observation showed certain correlations between the domain patterns and the remagnetization curve shapes. The main difference between the remagnetization curves for the two asymptotic cases of the nucleation-dominated and wall-motion-dominated remagnetization lies in their curvatures. Characteristic for these two cases is the opposite sign of the second derivative of the remagnetization curve. In order to suppress the undisturbed wall motion and hence to reduce the role of the domain wall motion as compared with the nucleation in the magnetization reversal process a high rate sputtering during the film manufacturing was applied. Fig. 2 shows the time dependent remagnetization curves and domain patterns of two samples with different domain structures and different remagnetization character. The lower half of the figure corresponds to the case with stronger wall pinning and more pronounced nucleation processes.

\section{MICROMAGNETICS}

The micromagnetic model describing the remagnetiza-
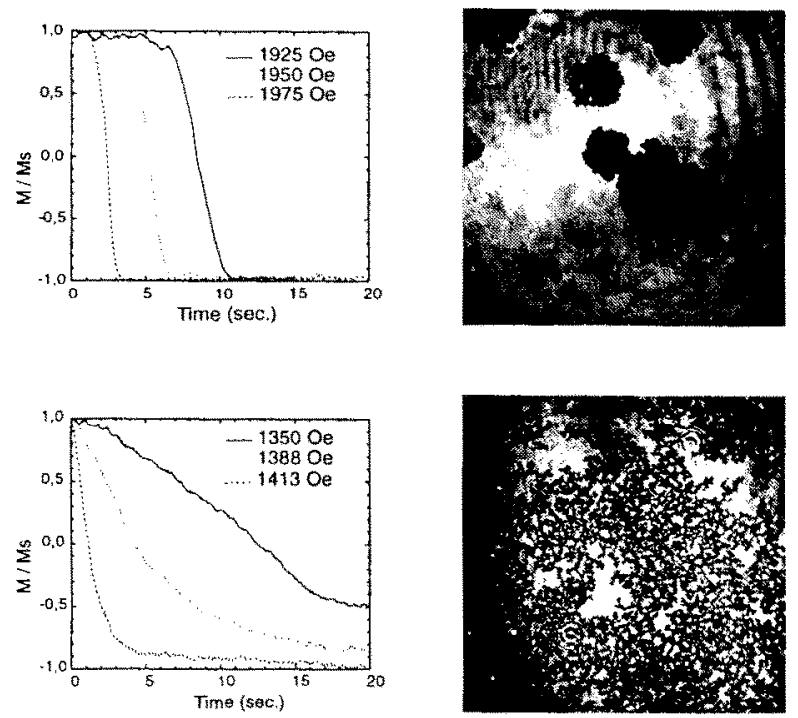

Figure 2: Time dependence of the Kerr amplitude during the magnetization reversal from one saturated state into another, measured for a sample with circular-like domain patterns and wall motion dominated reversal behavior (left) and for a sample showing dendritic domain structure with dense nucleation (right).

tion process includes the external field effect, the magnetostatic (dipole-dipole) coupling and the exchange coupling. The corresponding energy terms:

$$
\begin{gathered}
E_{Z}=-\vec{H} \cdot \vec{m}_{i}, \\
E_{i j}^{d}=\frac{1}{r_{i j}^{3}}\left[\vec{m}_{i} \cdot \vec{m}_{j}-\frac{3}{r_{i j}^{2}}\left(\vec{m}_{i} \cdot \vec{r}_{i j}\right)\left(\vec{m}_{j} \cdot \vec{r}_{i j}\right)\right], \\
E_{e x}=-2 J \vec{S}_{i} \cdot \vec{S}_{j}
\end{gathered}
$$

are written for elementary cells on a two-dimensional hexagonal grid. $\vec{m}_{i}=V \vec{M}_{i}$ is the magnetic moment of the $i$ 'th cell with the volume $V$ and magnetization $\vec{M}_{i}$ while $r_{i j}=\left|\vec{r}_{j}-\vec{r}_{i}\right|$ is the distance between two arbitrary cells $i$ and $j$. The exchange coupling between two spins $\vec{S}_{i}$ and $\vec{S}_{j}$ (3) is actually a quantum mechanical coupling, which is manifested through the existence of the wall energy $\sigma_{W}$.

Considering the case of strong anisotropy the energy contribution of one single cell can be reduced to the following scalar form:

$$
E_{i}=-\frac{1}{2} l d \sigma_{W} \sum s_{i} s_{j}+m^{2} \sum \frac{s_{i} s_{j}}{r_{i j}^{3}}-H m s_{i}
$$

Here $s_{i}$ stands for the magnetization state of the $i$ 'th cell, which can take values " 1 " and "-1" corresponding to the "up" and "down" magnetization directed perpendicular to the film surface.

The sums in (4) run over all cells in the corresponding coupling range, hence the first sum must be taken over 


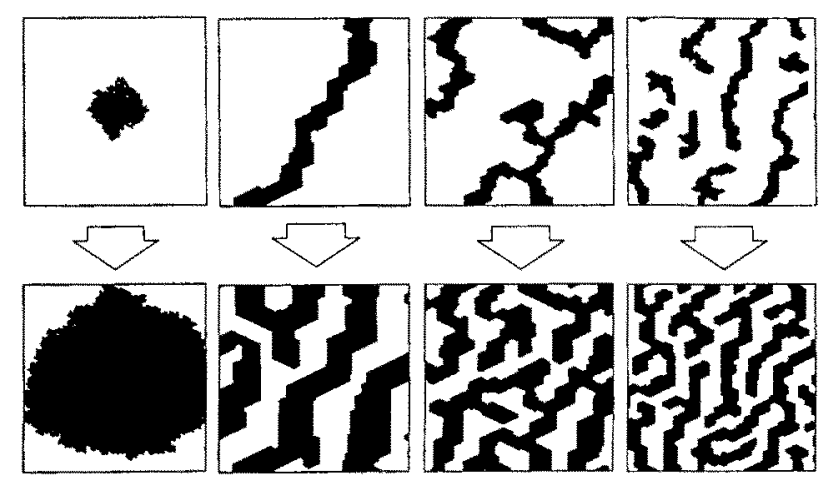

Figure 3: Domain patterns in the nearly demagnetized state (below) and in an intermediate state calculated for four different samples with different values of the saturation magnetization $M_{S}=0.1 H_{0}, 0.7 H_{0}, 1.0 H_{0}, 1.5 H_{0}$ (from left to right), where $H_{0}$ is the effective exchange field (7). The area of each square is equal to $10 \mu \mathrm{m} \times 10 \mu \mathrm{m}$.

all neighboring cells while the range of the second sum is practically unlimited because of the long-range character of the magnetostatic interaction. Although (4) gives a basis for carrying out Monte Carlo simulations the calculations were done in terms of the effective field [7], representing a computationally somewhat more efficient method. The effective field in the discrete case is defined as follows:

$$
H_{i}^{\text {eff }} \stackrel{\text { def }}{=}-\frac{\Delta E_{i}}{\Delta m}=-\frac{E_{i}}{m}
$$

Then the Monte Carlo condition controlling whether the remagnetization takes place or not will be rewritten as a condition for the effective field, that gives the following formula for iterative calculation:

$$
s_{i}^{t+1}=\operatorname{sign}\left[\sum s_{j}^{t}+\frac{H_{a}+H_{d}^{(i)}}{H_{0}}-\Delta_{i}\right]
$$

$H_{a}$ is the external magnetic field and $H_{d}^{(i)}=m \sum s_{j}^{t} / r_{i j}^{3}$ with $s_{j}^{t}$ standing for the state of the $j^{\prime}$ th cell at the $t^{\prime}$ th actualization step gives the demagnetizing field, the exact calculation of which in general is a challenging computational problem. The FFT algorithm developed here after the method proposed in [8] gives a very efficient way of calculating $H_{d}$. The function sign truncates its argument to " 1 " or " 1 ", giving in this way the transition function we were looking for. $\Delta_{i}$ is the random number array containing both thermal and local fluctuations. In addition an abbreviation $H_{0}=l d \sigma_{W} / 2 m$ has been introduced which is regarded as an effective exchange field. Here $d$ is the film thickness and $l$ is the length of the common boundary between two neigboring cells. Using the known expressions for the Bloch wall energy $\sigma_{W}=4 \sqrt{A K_{U}}$ and the wall thickness $\delta_{W}=\pi \sqrt{A / K_{U}}$ the expression for the effective exchange field introduced above can be reduced to the

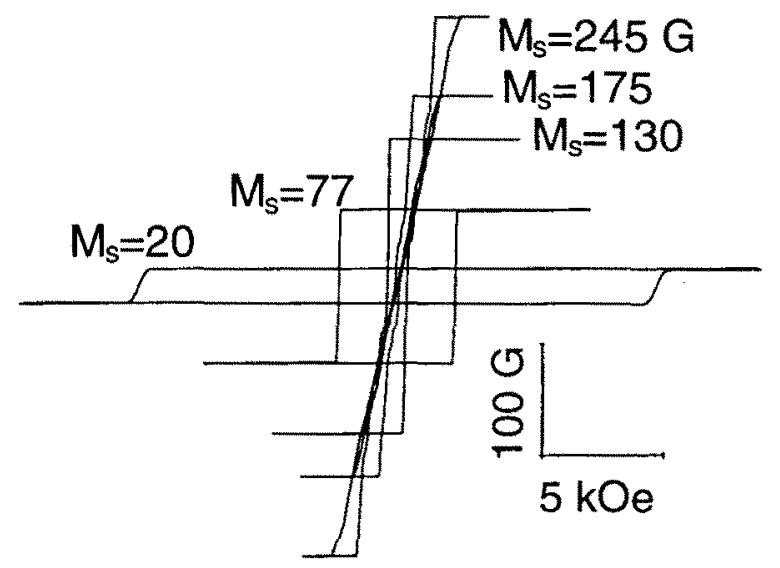

Figure 4: Hysteresis loops calculated for different values of $M_{S}$ taking $K_{U}$ equal to $3 \cdot 10^{6} \mathrm{erg} / \mathrm{cm}^{3}$ in accordance to the experimental measurements.

following form:

$$
H_{0}=\frac{l d \sigma_{W}}{2 m}=\frac{\pi d D \sqrt{A K_{U}} / \sqrt{3}}{2 M V} \approx \frac{H_{k}}{10 \kappa}
$$

Here $V$ is the cell volume. $H_{K}=2 K_{U} / M_{S}$ is the anisotropy field and $\kappa=D / \delta_{W}$ is the ratio between the elementary cell size and the domain wall width.

The model allows us to calculate both the domain patterns and the hysteresis loops. Fig. 3 shows some patterns calculated for different values of the saturation magnetization $M_{S}$. Approaching the compensation point the saturation magnetization of the sample and hence the magnetostatic interaction energy becomes smaller and the domain wall energy minimization begins to control the domain dynamics. The model developed here contains actually only one fitting parameter $\kappa$, characterizing the size of the elementary cell. All other quantities, like $M_{S}$ and $K_{U}$, can be measured experimentally. In the present calculations $\kappa=10$ was chosen corresponding to the cell size of 0.1 $\mu \mathrm{m}$.

Fig. 5 shows the perpendicular component of the demagnetizing field calculated for different domain patterns. One sees that the fine stripes lead to an efficient suppression of the demagnetizing field, and hence its energy, while the broad circular domains cause the field reduction actually only in the regions close to the domain boundaries.

One of the critical topics of micromagnetic simulations in general is the proper description of the time dynamics. Although there is no absolute time calibration done in this model one can get some useful information about the temporal dynamics with respect to a relative time scale, given by the number of the actualization steps during the calculation. Using this method of calibration, its possibility being simply postulated here, to each computational step a real time step $t_{0}$ can be assigned. The correlations between domain patterns and the dynamic remagnetization curves (Fig. 

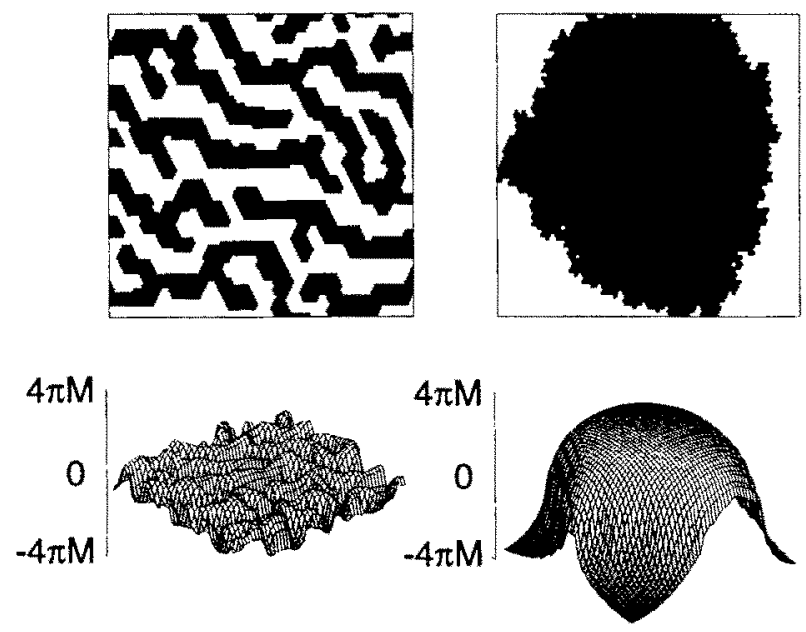

Figure 5: The domain patterns and perpendicular component of the demagnetizing field (averaged over the sample thickness) calculated for different values of the saturation magnetization $M_{S}$ (see Fig. 3).

6) calculated in this way show good agreement with the experiments (Fig. 2).

\section{CONCLUSIONS}

The magnetic properties of ferrimagnetic amorphous $\mathrm{Fe}_{1-x} \mathrm{~Tb}_{x}$ thin films and the domain patterns observed in these films using a Kerr-microscope showed strong dependence on the film composition in the whole range of the compositional parameter $0.16<x<0.35$.

Both experimental and micromagnetic studies showed the important role of the long range dipole-dipole interaction in the formation of different domain patterns. This result is consistent with other experimental results, as well as Monte Carlo simulations of Lyberatos et al $[6,9]$.

The FFT method of calculating the demagnetizing field proved to be a very useful tool, revealing such features of the demagnetization field effect which can not be adequately described in the framework of the mean field approximation or other truncation methods $[6,9]$.

The micromagnetic simulations showed that all domains are intrinsic stripe domains growing almost circularly only till a certain size, which than determines the domain stripe width. The spatial inhomogeneities as well as the thermal fluctuations distort the unperturbed domain growth resulting in irregular stripe branchings and random changes in the domain growth direction. The so called "cauliflower" domains must represent an unstable mode of domain growth, which appears only in the case of a certain subtle balance between the ordering influence of the demagnetizing field and the disordering effect of inhomogeneities and fluctuations.

Further investigations of this type in the vicinity of the compensation point seem to be very important, since the
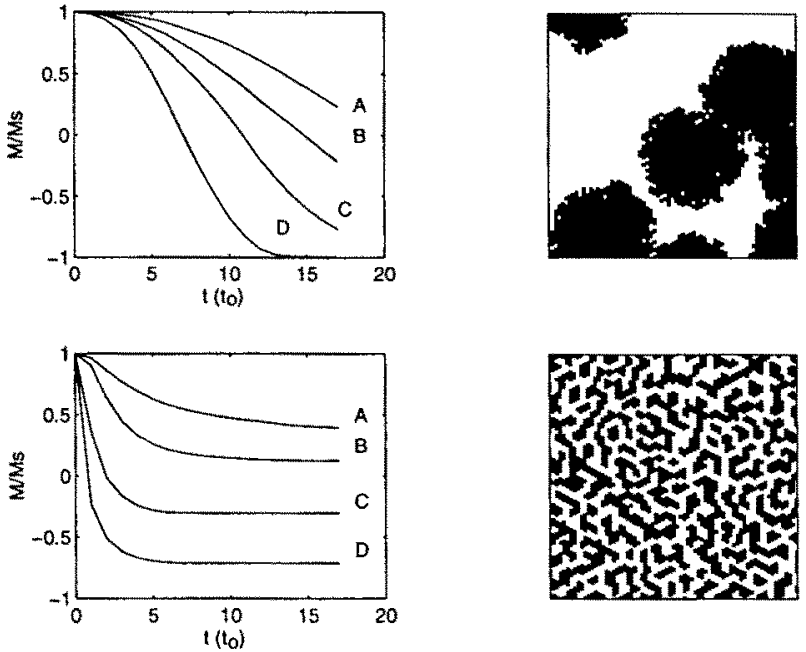

Figure 6: The remagnetization curves and domain patterns in the case of the wall-motion-dominated reversal for $M_{S}=$ $0.1 H_{0}$ at constant external field $H_{\alpha}: \mathrm{A}, H_{a}=5 H_{0} ; \mathrm{B}$, $H_{a}=6 H_{0} ; \mathrm{C}, H_{a}=6.7 H_{0} ; \mathrm{D}, H_{a}=6.8 H_{0}$ (above) and in the case of the nucleation-dominated reversal calculated for $M_{S}=2 H_{0}: \mathrm{A}, H_{a}=0.8 H_{0} ; \mathrm{B}, H_{a}=0.85 H_{0} ; \mathrm{C}$, $H_{a}=H_{0} ; \mathrm{D}, H_{a}=1.2 H_{0}$ (below).

magnetostatic effects become negligible in this case and the observed domain structures are of more intrinsic nature, reflecting the morphology and the atomic structure of the film as well as the effect of the thermal fluctuations.

Such investigations can be therefore very helpful in understanding the physical limitations of the micromagnetic calculations and in gaining more insight in the microscopic mechanisms of the magnetization reversal as well.

\section{REFERENCES}

[1] M. Mansuripur: The Physical Principles of MagnetoOptical Recording, Cambridge University Press, 1995

[2] H. Rohrmann and H. Hoffmann: Thin Solid Films, 175, 273 (1989)

[3] B. Lanchava et al: J. Magn. Magnet. Mat., in print (1997)

[4] K. Fabian, A. Kirschner, W. Williams, F. Heider, A. Hubert, and T. Leibl: Geophys. J. Int, 124, 89 (1993)

[5] Xiao Hu and Y. Kawadzoe: Phys. Rev. B, 49, 3294 (1994)

[6] A. Lyberatos, J. Earl, and R. W. Chantrell: Phys. Rev. B, 53, 5493 (1996)

[7] W.F. Brown: Micromagnetics, John Wiley \& Sons, New York, 1963

[8] M. Mansuripur and R. Giles: Computers in Phys., 4, 291 (1988)

[9] L.C. Sampaio, M.P. de Albuquerque, and F.S. de Menezes: Phys. Rev. B, 54, 6465 (1996) 